\title{
Diabetic oxidative stress and bone loss complications
}

\author{
Orlando L. Catanzaro ${ }^{1}$, Nicole Brasquet ${ }^{1}$ and Alejandra Arganaraz ${ }^{1,2}$ \\ ${ }^{1}$ Dental and Medical School -AOA Univ. Del Salvador,
}

${ }^{2}$ Department of Physiology -Univ. Argentina John F. Kennedy.

Corresponding Author: Orlando L. Catanzaro, Dental and Medical School -AOA Universidad Del Salvador, Facultad de Ciencias Médicas, Escuela de Odontologia, Cátedra de Fisiología General y Neurofisiologia. Buenos Aires, Argentina.

Received date: October 30, 2020; Accepted date: November 16, 2020; Published date: March 06,2021

Citation: Orlando L. Catanzaro, Brasquet N. and Arganaraz A., (2021) Diabetic oxidative stress and bone loss complications, J. Endo and Disor 5(1); DOI:10.31579/2640-1045/056

Copyright: ( 2021 , Orlando L. Catanzaro, This is an open access article distributed under the Creative Commons Attribution License, which permits unrestricted use, distribution, and reproduction in any medium, provided the original work is properly cited.

\begin{abstract}
Diabetes mellitus is a group of metabolic disorder characterize by and absolute or partial insulin deficiency. Diabetic hyperglycemia is produce by the effect of homeostasis between proteolytic enzymes, their inhibitors and the antioxidants defense that protect and repair vital tissues and molecular components. Bone consist of both component and trabecular bone tissue. Organic matrix and albumin form part of noncollagenous of bone .Initiation of mineralization and collagen fibrils form the phase of mineral matrix. Calcium flux into and out of bone depend of osteoclastic and osteoblastic activity. The remodeling is initiated by resorption and new bone formation at the resorption site. Diabetic complication is a critical factor for bone pathology and could start early inflammatory stage even before hyperglycemia. Diabetic produces bone loss from reduce osteoblast activity. Partly insulin deficiency produce defective bone remodeling indirect by oxidative stress. The current treatment for defective bone in diabetes state include biophosphonate and cinaciguat. Biphosphonate inhibit bone resorption, but may worsen bone quality. A novel type of activation of sGMP is cinaciguat an NO independent activator of oxidative GC, increase c GMP synthesis on diabetic and restore proliferation and survival of osteoblasts. Chronic hyperglycemia interferes with the oseointegration of implants in diabetics. Both diabetic and aging plays a role in abnormal differentiation of osteroblasts. In diabetic patients may improve the oral health to have a positive impact if optimal glycemic control is emphasized. However with cinaciguat present as a novel paradigm enhancing bone formation under hyperglycemia and protect bone implants.
\end{abstract}

Keywords: diabetes mellitus; metabolic disorder; hyperglycemia; osteoclastic; osteoblastic activity

Diabetic mellitus is a group of metabolic disorders characterized by an absolute or partial insulin deficiency [1]. There are 4 types of diabetes: (i) type 1 diabetes, caused by destruction of pancreatic $\beta$-cells; (ii) type 2 diabetes, which is at least $90 \%$ of cases (occurs as a result of progressive defect in insulin secretion;(iii) gestational diabetes (occurs In the second or third trimester of pregnancy); and (iv) diabetes of other causes pancreatic anomalies, (eg. pancreatic fibrosis, drugs or chemical -induced or organ transplantation) [2]. A loss of homeostatic balance between proteolytic enzymes (neutrophil elastase) and their inhibitors (alfa $1^{-}$ antitrypsin) and reactive oxygen species (ROS) and the antioxidant defense that protect and repair vital tissue, cell, and molecular components is believe to be responsible for the effect of diabetic hyperglycemia. The results from bone loss with the changes in remodeling during normal aging may be accelerated by extrinsic and intrinsic factors. Although bone appears inert, it is a dynamic tissue that receives about $10 \%$ of the cardiac output and remodels throughout life [3]. Bone consist of both compact and trabecular bone. The extracellular bone components include a solid mineral phase associated with 90-95\% of organic type 1 and collagen matrix. Noncollagenous is part of organic matrix and contains albumin, cell attachment/ signaling proteins as osteopontin and fibronectin, calcium binding proteins and osteocalcim, biglycan and decorin. The proteins of serum are responsible for the organization of collagen fibrils, initiation of mineralization and the mineral phase to the matrix. The skeleton contains over $99 \%$ of the calcium in the adult body. There are high rates of calcium flux into and out of bone, mediated by osteoblastic and osteoclastic activity, the 0.5-1$0 \%$ of exchangeable calcium is within then extracellular fluid.

Calcium channels can be activated by hormones, metabolites or neurotransmitters affecting the $50 \%$ of total serum calcium that is ionized.

The ionized calcium is concentrated in the extracellular fluid by across renal and intestine epithelia mediated by the effect of parathyroid hormones, $\mathrm{D}_{3}$ vitamin and calcium. In the control of calcium and phosphorus homeostasis, evidences has been demonstrated that vitamin $\mathrm{D}_{3}$ and is synthetic analogs play a multiple functional role in the enhancement of antimicrobial peptide, expression and immunomodelatory actions $[4,5]$. Current evidence indicates that Vit. D mediate activation and regulate genes that mediate osteoblast and osteolast functions [6]. Healthy bone models and remodels occurs throughout life as a dynamic effect of bone tissue. The skeleton increases in size in response in the stresses placed upon it. This involves new bone formation that is independent of tissue resorption and in this form can assume a new shape or cortical thickening. The remodeling is initiated by resorption and by new bone formation in the same place of resorptive site. Mechanical bone stresses are transmitted to osteoclast or osteoblast or to their precursors by osteocytes. Bone resorption reflex the sum of osteoclast recruitment and death, and the relation of the average cell degrades matrix [7]. Diabetes mellitus is associated with overproduction of inflammatory cytokines and oxidative stress in response to the state of 
hyperglycemia $[8,9]$, and interleukin- $1 \beta$ and tumor necrosis factor (TNFalfa) that leads to the destruction of pancreatic $\beta$-cells [10]. Diabetic complications, including bone pathology, could start in develop the early inflammatory stages of the disease, even before establish the hyperglycemia and /or the glucosuria based diagnosis for diabetes [11]. Bone loss are frequent complications of diabetes [1]. Bone loss in diabetes type 1 is mainly from reduced osteoblast activity, thus, current osteoporosis therapies aimed at inhibiting osteoclast are inadequate [12, 13]. In diabetes type 2 bone formation decreased and increase facture risk, but bone mineral density may normal or increased, while bone quality is impaired [14]. Inflammatory processes are well known to interact with remodeling of bone tissue in the vicinity, causing bone enhanced rate of bone resorption and new bone formation with the former usually being dominant $[15,16]$. Pro-inflammatory mediators formed by the kallikreinkinin system can stimulate bone resorption and synergistically potentiate bone resorption induced by IL-1 $\beta$ and TNF-alfa. Bone loss due to increased number of activity resorbing osteclasts observed in periodontal disease and rheumatoid arthritis and diabetes is usually considered being caused by excess of cytokines stimulating osteoclast genesis compared to those with inhibitory effects. Stimulatory cytokines include interleukin1(IL-1),IL-6,IL-11, IL-17, leukaemia inhibitory factor(LIF), oncostatin $\mathrm{M}(\mathrm{OSM})$, tumor necrosis factor (TNF-alfa) and transforming growth factor- $\beta$ (TGF- $\beta$ ), and the group inhibitory cytokines belongs IL-4, IL10, IL-13, interferon $-\Upsilon($ ITF- $\Upsilon$ ) $[17,18]$. Kinis can stimulate bone resorption in vitro $[19,20]$ and may more important synergistically potentiate the bone resorption effect of IL-1 an effect synergistic enhancement of prostaglandin biosynthesis [21]. Kinins have been known in a variety of biological effects including cardiovascular homeostasis inflammation, nociocepcion and bone resorption [22, 23, and 18]. Kinins are formed from kininogen either by plasma kallikrein, which is activated early in the coagulation cascade, or tissue kallikrein, which is activated by proteases at injured sites [24]. Their mediate biological effects by acting on two types of receptors namely $\mathrm{B}_{1}$ and $\mathrm{B}_{2}$. Molecular cloning of the bradykinin $\left(\mathrm{BKB}_{1}-\mathrm{R}\right) \mathrm{B}_{1} \mathrm{R}$ receptors and the $\mathrm{B}_{2} \mathrm{R}$ receptors $\left(\mathrm{BKB}_{2}-\right.$ $\mathrm{R})$ from a variety of species including humans, revealed that they belong to the family of $\mathrm{G}$ protein-couple receptors [25]. The $\mathrm{BKB}_{2}-\mathrm{R}$ mediate many of the physiological effects of kinins are constitutively expressed and involved in the acute phase of the inflammation. The $\mathrm{BKB}_{1}-\mathrm{R}$, usually absent in normal tissues, are induced an over-expressed during tissue injury and following treatment with inflammatory mediators like endotoxin and cytokines. Furthermore the enhancement of $\mathrm{B}_{1}$ and $\mathrm{B}_{2}$ receptors expression and IL-1 $\beta$ and TNF- $\Upsilon$ was not only observed in the human osteoblasts cell but also in the intact mouse calvarial bones and primary culture of human gingival fibroblast [26]. Partly insulin deficiency produce defective bone remodeling in diabetes type 1 , but current evident indicates that hyperglycemia induce oxidative stress [27]. Increased reactive oxygen species (ROS) and decreased antioxidants defense mechanism contribute to diabetic tissue damage and bone loss $[28,29]$. Patients with type 2 diabetes have decreased bone formation and increased fracture risk, but bone mineral density may be normal or increased, ,while bone quality is impaired [30]. Compared with other complications, diabetic disease increase the osteoporosis in bone fragility and fracture [31]. Current experimental evidences suggest that durable hyperglycemia, the most remarkable character of diabetes, could impair bone regeneration and delay bone healing rates [32]. There is an increase of alveolar ridge reabsorption in edentulous patients with osteoporosis [33]. Greater alveolar crystal height loss is noted with osteoporosis and osteopenia [34]. Osteoporosis and osteopenia are associated to diabetes and periodontitis, attachment loss and gingival recession [35]. Induced bone mineral density was associated with increased clinical attachment loss [36]. On the other hand weak or not significant association between systemic bone mineral and clinical attachment loss was observed [37]. However an association between osteoporosis and clinical attachment loss provided stronger evidences [38]. In postmenopausal women was found that periodontal disease and osteoporosis was associated with lower vitamin D and higher concentrations of RANKL and osteoprotegerin [39]. Between others, reduced bone mineral density is a critical risk factor for periodontitis rather than a casual factor between periodontal disease and reduced bone mineral density. As the natural teeth are more prone to loss and, as well also, osteoporosis, in diabetic periodontitis, two components to ameliorate all adverse effect of periodentits on bone can be describe. The current medications for osteoporosis include calcium, vitamin D, parathyroid hormone, selective estrogen receptor modulators, calcitonin, bisphosphonates and cinaciguat. With increasing longevity, it is important to remember that osteoporosis in diabetes is common to both sex. Clinical therapy for osteoporosis is a lifelong - intervention. Diabetic hyperglycemia could delay bone healing rates, which is critical for the osseointegration of dental implants. Bisphosphonates are analogs of inorganic pyrophosphates. Biophosphonates inhibit bone resorption, improve bone mineral density but may worsen bone quality by repressing bone formation. There are differences in the chemical and, physical structure of bisphosphonates, and these differences may explain variations in clinical observations, as potency at enzymatic level, binding affinity, distribution accumulation and release. Bisphosphonates of low affinity do not bind as tightly on the surface of the bone for removal by osteoclasts and the drug comes off easily from bone and its effect may shorter in duration. Bisphosphonate is derived from the base of the drug, with two phosphonate (PO3) groups covalent linked to a central carbon. The carbon atom confers resistance to hydrolysis with two side chains. The short side -chain usually has a hydrolysis moiety and provide a strong affinity for calcium crystal and bone mineral. The long- side -chain determines the mode of action and the strength of the bisphosphonate. Bisphosphonates have low intestinal absorption and are excreted via renal. Bisphosphonates inhibition osteoclasts depend by two mechanisms: if long side -chain contains or not nitroside groups. When lack a nitrogen in the side-chains are metabolized by osteoclasts, interfere with osteoclasts and induce apoptosis. On the other hand the side with nitrogen contains side groups reduced osteoclasts recruitment and induced osteoblasts to produce an osteoclast inhibiting factor. In experimental animals, bisphosphonate (alendronate) has been showed to inhibit repair of normal micro damage, so micro damage accumulation may occur [40]. To date bisphosphonates trials, specific gains in bone quality architecture, turnover, damage accumulation and mineralization have not been reported in human maxilla or mandibule. As a matter of fact hyperglycemia induces oxidative stress and loss bone mineral density and reduce bone mass. These two factors could impair bone regeneration which is critical for the osseointegration implants in patients with diabetes [41]. More effective methods in reverse the bone loss in diabetes could be an important factor. As a vital molecule NO can diffuse into target cells and activate intracellular receptor, the soluble guanylate cyclase (sGC). Cyclic guanosine monophosphate -5 (cGMP) is generated by cytoplasmic sGC and hydrolyzed by phosphodiesterase -5 (PDE5) which stimulates protein kinase $\mathrm{G}(\mathrm{PKG})$, and produce a series of biological effects as reversing oxidative stress, inducing osteoblast generation [42]. In diabetic conditions existing evidences that the cGMP/PKG II could be disturbed [43], such as increased expression of PDES and decreased bioavailability of cGMP [44] soluble guanidine cyclase (sGC) drug have been discovered to resist interfered cGMP/PKG II pathway. A novel type of activator of sGC is Cinaciguat (Bay 58-2667), an NO-independent activator of oxidative guanylate cyclase increase cGMP synthesis under diabetic conditions and restore proliferation, differentiation, and survival of osteoblasts. The effect of the drug increase trabecular and cortical bone in type 1 mice by improving bone formation and osteoblasts. In type 1 diabetes bone loss is due from reduced osteoblast activity. On the other hand defective bone remodeling in diabetes is partly due to insulin deficiency. Insulin requires NO/cGMP/PKGII signaling for proliferative effect in osteoblasts. Under hyperglycemia conditions decrease insulin activation of the NO/cGMP/PKGII in osteoblasts expose to high glucose 
.Restoring cGMP synthesis reduces oxidative stress, recovers osteoblast functions, decreases osteocyte apoptosis, and prevent bone loss in the insulin -deficient mice. According to Joshua et al [45] cinaciguat produce a transient decline in systolic pressure. Research shows that cinaciguat could improve cardiac dysfunction in type 1 diabetic rats [46], which indicated that it may play a protective role in glucotoxicity environments. Have been demonstrated that cinaciguat could reverse the damage of osteoblasts caused by high glucose and protect the osteogenic function in TID mice [47]. Osseointegtration is a combination of living bone tissue and implants and the first stage of bone implants interactions is essential for the formation of osseointegration interface. It is difficult to explain the molecular effects of cinaciguat on osteointegration under hyperglycemia condition. Studies in diabetic rats (Zucker or STZ) showed that chronic hyperglycemia interferes with the osseointegration of implants by determining expression of fibronectin and integrin alpha 5-beta -1[48]. Advanced glycation end products accumulate in body tissues with the age's progression, and under hyperglycemic conditions inhibit osseointegration and compromise the biochemical properties of the bone implant interface. Persistent hyperglycemia plays a role in abnormal differentiation of osteoblasts, making bone tissue more susceptible to resorption [49]. Diabetes is associated with peri-implant soft tissue, crystal bone loss and even implant ailure. Implant survival and primary stability parameters in diabetes conditions varying with hemoglobin A1c, and increase in proportion with the rising hemoglobin A1c levels. Periimplant plaque index, bleeding on probing depth and crest bone loss are significantly higher in diabetic than in control. In diabetic patients may improve the oral health to have a positive impact if optimal glycemic control is emphasized. No/cGMP/PKG pathways activator with cinaciguat present a novel paradigm for enhancing bone formation under conditions of hyperglycemia and oxidative stress.

\section{References}

1. American Diabetes Association. (2018) Classification and diagnosis of diabetes standards of medical care in diabetes. Diabetes Care 45(Suppl.1): S 13 -s 27.

2. American Diabetes association (2018) Classification and diagnosis of diabetes. 39(Suppl.1): S13-s22.

3. Lindsay R, Cosman F. (2006) Bone structure and metabolism. Chapter 333.Harrison's Ingternal Medicine. New York: Mc Graw -hill Co.

4. Wang TT, Nestel FP, Bourdeau V, et al. (2004) Cuting edge 1, 25dihydroxyvitamint D3 is a direct induced of antimicrobial peptide gene expression. J. of Immunol.173:2909-2912.

5. Panda DK, Miao D, Tremblay MÍ. (2001) Targeted ablation of the 25-hydroxyvitamin D alpha-hydolaxylase enzyme evidence for skeletal reproductive and immune dysfunction. Proc Nat Acad Sci USA 98: 7498-7503.

6. Pike JW, (2005) Molecular action of vitamin D and its analogs innttranscriptional regulation in vitro. Program and abstracts of the Endocrine Society's $87^{\text {th }}$ annual Meeting S13-S20.

7. Ott SM. (1996) Principles of bone biology. Edited by Bilezkian IP ed. San Diego, CA: Acad Press 18:231.

8. Ravinovitch A, Suarez-Pinzon WL. (1998) Cytokines and their roles inpancretic islet beta-cell destruction and insulin -dependent diabetes mellitus. Biochem Pharmacol 55:139-149.

9. Yemeni KK, Bai W, Khan BV et al. (1999) Hyperglycemia induced activation and nuclear transcription factor kappaB I vascular smooth muscle cells. Diabetes 48:855-864.

10. Mandrup-Poulsen T. (1996) The role of interleukin-1 in the pathogenesis of IDDM. Diabetology 39: 1005-1020.

11. Zuccollo A, Navarro M, Frontera m, Cueva F, Catanzaro O. (1999) The involvement of kalikrein-kinin system in diabetes type 1 (insulitis) Immunopharmacology 45:69-74.
12. Selmeyer D E, Civielli R, Hofbauer LC, Khosla S et al. (2016) skeletal metabolism, fracture risk and fracture ourcomes in type and type 2 Diabetes. Diabetes 65:1757-1766.

13. Hygun K, Stanup-Linde J, Hofbauer T, Vestergaard P, et al. (2017) Mechanisms n endocrinology diabetes mellitus: a state of low bone turnover - a systemic review and meta-analysis. Eur J Endocrinol, 176:R137-R157.

14. Napoli N, Chandran M, Pierroz DD, Abrahamsen B ,et al. (2017) Bone and Diabetes Working Group. Mechanims of diabetes mellitus -induced bone fragility. Nar Rev Endocrinol. 13:208219.

15. Walsh NC, Gravallese EM. (2004) Bone loss in inflammatory arthritis: mechanism and treatment strategies. Curr.Opin Rehumatol 16:419-3427.

16. Lerner UH. (2006) Inflammation induced bone remodeling in periodontal and the influence of postmenopausal osteoporosis. $J$ Dent Res 85:596-607.

17. Firestein GS. (2001) Evolin concepts of rheumatoid arthritis. Nature 423:356-361.

18. Horowitz J, Lorenzo JA. (2002) Local regulators of bone IL1TNF, lymphotoxin, interferon $\Upsilon$, IL-8, IL-10, IL-4, the lift/IL-6 family and additional cytokines. I Bilezikian JP, Raizs IG, Roldan GA. Ext. Priciples of bone Biology. ${ }^{\text {nd }}$.ed. San Diego Acad. Press p.961-978.

19. Lerner2 UH, Lundberg P. (2002) Kinis and neuro-osteointegration factors .In Bilezikian JP, Raisz LG, Roldan GA. Eds. Principles of bone Biology. $2^{\text {nd }}$ ed. San Diego. Acad. Press. p.773-799.

20. Lerner UH. (1991) Bradykinin synergistically potentiates IL-1 induced bone resorption and prostanoid biosynthesis in neonatal mouse calvarial bones. Biochem Biophys Res Commun, 175:775783.

21. Bretcher AB, Lerner UH. (2007) Bradykinin potentites cytokine induced prostaglandin biosynthesis in osteoblasts by enhanced expression of COX-2 resulting in increased RANKL. Arthritis Rheum 56:910-953.

22. Calixto JB, Cabrini DA, Ferreira J, (2000) Campos MM. Kinins in pain and inflammation. Pain 87:1-5.

23. Marceau F, Bachvarov DR, (1998) Kinin receptors. Clin Rev Allergy Immunol, 16:385-401.

24. Bhoola KD, Figueroa CD. (1992) Bio regulation of kinins, kallikreins, kininogens and kininases. Phamacol Rev, 44: 1-80.

25. Hess JF, Derrick AW, Mac Neil T, Borkowski JA. (1996) The agonist selective of a mouse $\mathrm{B}_{1}$ bradykinin receptor differs from human and rabbit $\mathrm{B}_{1}$ receptor. Inmmunopharmacology 33:1-8.

26. Bernhold Brechter A, Peterss E, Lundgren I, Lerner UH. (2008) Kinin B1 and B2 receptor expression in osteoblasts and fibroblasts in enhanced by interleukin -1 and tumor necrosis factor -alfa. Effects dependent on activation of NF-kB and MAP kinase. Bone 43:72-83.

27. Kalaitzoglou e, Popescu I, Bunn RC, Fowkles JL, et al. (2016) Effects of type 1 diabetes on osteoblats, osteocyts, and osteoclasts. Curr Osteoporos Rep, 14:310-319.

28. Hamada Y, Kitazawa S, Kitazawa R, Fujii H et al. (2007) Histomorphometric analysis of diabetic osteopenia in streptozotocin -induced diabetic mice a possible role of oxidative stress. Bone 40:1408-1414.

29. Hamada Y, Fuii H, Kitazawa R, Yodoi J, Kitazawa S et al. (2009) Thioredoxin -overexpression in transgenic mice attenuates streptozotocin -induced diabetes osteopenia a novel role of oxidative stress and therapeutic implications. Bone 44:936-941.

30. Giacco F, Brownlee M. (2010) Oxidative stress and diabetes complications. Cir Res, 107:1058-1070.

31. Aguilar-Salvatierra A, Calvo-Guisado JL, Gonzalez-Jaranay M Moreu G et al. (2016) Peri-implants evaluation of immediately 
implants place in esthetic zone in patients with diabetes mellitus type 2; a two years study. Cli Oral Implants res 27:156-161.

32. Hamann C, Geettsch C, Mettelsiefen J, Henkenjohann V, et al. (2011) Delayed bone regeneration and low bone mass in ragt model of insulin -resistant I type 2 diabetes mellitus is due to impaired osteoblast function. Am J Physiol Endocrinol Met 301: F 1220-1228.

33. Rubin MP, Patsch JM. (2016) Assessment of bone turnover and bone quality in type 2 diabetic bone disease: current concepts and future direction. Bone res 4: 16001.

34. Devlin h, Fergunson MW, (1991) Alveolar ridge resorption and mandibular atrophy .A review of the role of local and systemic factors. Br Dent $J$ 170:101-104.

35. Catanzaro OL, Andornino A, Brasquet N, Di Martino I, Arganaraz A. (2018) The effect of gingival aging in diabetic and non-diabetic status. An experimental study. Acta odont lat 31:32-36.

36. Mohammad AR, Hooper DA, Vemilyea SG, Mariotti A, et al. (2003) An investigation of the relationship between systemic bone density and clinical periodontal status in post-menopausal Asian-American women. Int Dent J, 53:121-135.

37. Pilgram TK, Hildebolt CT, Dotson M, Cohen SC, et al. (2002) Relationship between clinical attachment level and spine and hip bone mineral density :data from healthy postmenopausal women. J Perodotol 73:298-301.

38. Shum I, Leung PC, Kwok A, Corbet EF, et al. (2010) periodontal conditions in elderly men with and without osteosporosis or osteopenia. J Periodontal, 811396-1402.

39. Jabbar S, Drury J, Fordham J, Datta HK, et al. (2011) Plasma vitamin $\mathrm{D}$ and cytokines in periodontal disease and postmenopausal osteoporosis. J Peridontal Res, 46:97-104.

40. Li j, Mashiba T, Burr DB. (2001) Bisphophonate treatment suppresses not only stochastic remodeling but also targeted repair of micro damage. Cacif Tissue Int, 69:281-286.

41. Catalano DL, Brittem TM, Storch DL, Calderon NL, et al. (2013) Hyperglycemia induced an intrinsic alterationin type 2 diabetes derived osteosclast function. Oral Dis, 19:303-312.

42. Wang L, Chopp A, Szalad C, Liu M et al. (2011) Phosphodiesterase -5 is a therapeutic target for peripheral neuropathy in diabetes mice. Neuroscience 93:399-410.

43. Craven PA, Studer RK, De Rubertis FR, (1994) Impaired nitric oxide -dependent cyclic guanosine monophosphate generation in glomeruli suppression of cholinergic response. J Clin 93:311-340.

44. Huynh K, Bernardo BC, Mc Mullen JR, Ritchie RH. (2014) Diabetic cardiomyopathy: mechanism and new treatment strategies targeting antioxidant signaling path ways. Pharmacol Ther 142: 345-415.

45. Joshua j, Rasgaswami H, Kalyanaraman H, et al. (2014). Soluble guanilato cyclase as a novel treatment target for osteoporosis. Endocrinology 155:4720-4730.

46. Matyas, Nemeth BT, Olah A, Hidi L et al. (2015). The soluble guanylate cyclase activator cinaciguat prevents cardiac disfunction in a rat model of type 1 diabetes mellitus. Cadivasc Diabetology, 14:p.145.

47. Kalyanaraman H, Schwaerzer G, Ramdam G, Castillo F et al. (2018). Protein kinase G activation reverse oxidative stress and restore osteoblast and bone formation in male mice with type 1 diabetes. Diabetes 67:607-623.

48. Liuz, Zhou W, Liu s, XU X, et al. (2015). Potential mechanism for osseointegration of dental implants in Zucker diabetic fatty rats. Br Oral Maxillofac Surg, 53:748-753.

49. Quintero DG, Winger JN, Khashaba R, Borcke JL. (2010). Advance glycation endproducts and dental implant Osseointegration. J Oral Implantol, 36:97-103. 